\title{
Effect of Temperature and Maternal Age on Recombination Rate in Cattle
}

\author{
Botong Shen ${ }^{1 \dagger}$, Ellen Freebern ${ }^{1 \dagger}$, Jicai Jiang ${ }^{1,2}$, Christian Maltecca ${ }^{2}$, John B. Cole ${ }^{3}$, \\ George E. Liü and Li Ma ${ }^{1 *}$ \\ 'Department of Animal and Avian Sciences, University of Maryland, College Park, College Park, MD, United States, \\ ${ }^{2}$ Department of Animal Science, North Carolina State University, Raleigh, NC, United States, ${ }^{3}$ Animal Genomics \\ and Improvement Laboratory, BARC, USDA-ARS, Beltsville, MD, United States
}

\section{OPEN ACCESS}

Edited by:

Francisco Peñagaricano,

University of Wisconsin-Madison,

United States

Reviewed by:

Luiz Brito,

Purdue University, United States

Robert D. Schnabel,

University of Missouri, United States

${ }^{*}$ Correspondence:

LiMa

lima@umd.edu

tThese authors have contributed equally to this work and share first

authorship

Specialty section:

This article was submitted to Livestock Genomics,

a section of the journal

Frontiers in Genetics

Received: 19 March 2021

Accepted: 28 June 2021

Published: 20 July 2021

Citation:

Shen B, Freebern E, Jiang J,

Maltecca C, Cole JB, Liu GE and

Ma $L$ (2021) Effect of Temperature and Maternal Age on Recombination

Rate in Cattle.

Front. Genet. 12:682718.

doi: 10.3389/fgene.2021.682718
Meiotic recombination is a fundamental biological process that facilitates meiotic division and promotes genetic diversity. Recombination is phenotypically plastic and affected by both intrinsic and extrinsic factors. The effect of maternal age on recombination rates has been characterized in a wide range of species, but the effect's direction remains inconclusive. Additionally, the characterization of temperature effects on recombination has been limited to model organisms. Here we seek to comprehensively determine the impact of genetic and environmental factors on recombination rate in dairy cattle. Using a large cattle pedigree, we identified maternal recombination events within 305,545 three-generation families. By comparing recombination rate between parents of different ages, we found a quadratic trend between maternal age and recombination rate in cattle. In contrast to either an increasing or decreasing trend in humans, cattle recombination rate decreased with maternal age until 65 months and then increased afterward. Combining recombination data with temperature information from public databases, we found a positive correlation between environmental temperature during fetal development of offspring and recombination rate in female parents. Finally, we fitted a full recombination rate model on all related factors, including genetics, maternal age, and environmental temperatures. Based on the final model, we confirmed the effect of maternal age and environmental temperature during fetal development of offspring on recombination rate with an estimated heritability of $10 \%(S E=0.03)$ in cattle. Collectively, we characterized the maternal age and temperature effects on recombination rate and suggested the adaptation of meiotic recombination to environmental stimuli in cattle. Our results provided first-hand information regarding the plastic nature of meiotic recombination in a mammalian species.

\section{Keywords: recombination, maternal age, temperature, cattle, genetics}

\section{INTRODUCTION}

Meiotic recombination is an essential process that occurs in all sexually reproducing organisms. This process facilitates the pairing and alignment of homologous chromosomes during prophase, which leads to the formation of crossovers. These crossover events transfer genetic information between the maternal and paternal homologs, and in doing so, ensures that each offspring will

Abbreviations: SNP, single nucleotide polymorphism; Chr, chromosome; NOAA, National Oceanic and Atmospheric Administration; THI, Temperature humidity index. 
receive a unique combination of parental genomes. The extent and pattern of genetic reshuffling has important implications for evolution and population genetics, as well as breeding. However, errors in meiotic recombination can lead to aneuploidy, chromosomal abnormalities, and other deleterious outcomes (Hassold and Hunt, 2001; Lipkin et al., 2002). Thus, meiotic recombination must be well-regulated by cellular processes to prevent disturbances in the recombination pathway. It has been found that various factors influence meiotic recombination patterns in human and animal genomes. For instance, genomewide association studies (GWAS) have identified genes and genetic variants associated with recombination features in humans (Kong et al., 2008; Chowdhury et al., 2009), mice (Baudat et al., 2010), cattle (Sandor et al., 2012; Ma et al., 2015; Shen et al., 2018), and sheep (Johnston et al., 2016). Some of the genes from those studies, including RNF212, CPLX1, and PRDM9, have been reported to be associated with individual-level recombination rates across multiple mammalian species.

A variety of intrinsic and external factors affect recombination rates across individuals and populations. These factors can be derived from environmental conditions, such as temperature, or physiological and stressful conditions, such as starvation. The resulting changes in recombination rates pose benefits or consequences to the health and evolution of a species. Many studies have suggested that maternal age's intrinsic factor has a significant effect on recombination rate, but the direction of the effect is still debatable (Polani and Jagiello, 1976; Hussin et al., 2011; Martin et al., 2015). A recent multicohort analysis in humans reported a small but significant positive effect of maternal age on recombination rate, which contradicted previous studies in other human population (Martin et al., 2015). In mice and hamsters, a negative effect of maternal age was observed (Polani and Jagiello, 1976). However, no effect of maternal age was found for recombination rate in wild sheep (Johnston et al., 2016), but an increase was reported in swine (LozadaSoto et al., 2021). Also, extensive studies of the maternal age effect have been conducted in Drosophila, worms, plants, and yeast, but no consistent conclusion have been reached (Hunter et al., 2016; Modliszewski and Copenhaver, 2017). As for the paternal side, many studies reported no effect of paternal age on meiotic recombination (Griffin et al., 1995; Hussin et al., 2011). Although the biological reason remains unclear for the effect of maternal age on recombination, there are some proposed explanations for both directions of the effect. The positive effect can be explained by a selection hypothesis: the factors related to aneuploidies increase with maternal age, so eggs with more crossovers are more likely to overcome these and give a live birth in older mothers (Kong et al., 2004). The negative effect can be explained by another hypothesis that specific meiotic configurations are less likely to be properly processed with increasing maternal age, so recombination rate decreases with maternal age (Hassold et al., 1995).

Extrinsic factors, such as temperature and nutrient conditions, have also been found to influence meiotic recombination rates. In Drosophila, environmental stressors such as exposure to Ethylenediaminetetraacetic acid (EDTA) or nutritional deficiency were observed to increase the recombination rate (Levine, 1955).
Similarly, in the budding yeast, Saccharomyces cerevisiae, a lack of nutritional resources resulted in an increased recombination rate (Abdullah and Borts, 2001). However, the effect of temperature on meiotic recombination rates is more complicated. Some studies reported a positive correlation in Arabidopsis thaliana, Caenorhabditis elegans, and Melanoplus femurrubrum (Church and Wimber, 1969; Rose and Baillie, 1979; Francis et al., 2007), while other studies found a negative correlation in Allium ursinum (Loidl, 1989).

Additionally, both positive and negative correlations were detected in Drosophila (Stern, 1926). For instance, a recent study in Drosophila melanogaster reported a non-linear increase in meiotic recombination frequency in response to increased exposure to heat shock conditions (Jackson et al., 2015). This finding suggests that Drosophila can plastically modulate their recombination rate in response to environmental conditions, thus conferring greater adaptive potential to their offspring. In cattle, decreases in fertility rate have occurred due to the major factor of heat stress. In fact, Holstein cattle's conception rate in the summer season is $20-30 \%$ less than in the winter season (Cavestany et al., 1985).

The formation of the Animal Genomics and Improvement Laboratory (AGIL) has facilitated the development of genetic evaluations for economically important traits in United States dairy cattle. Such studies can enhance research to improve the health and efficiency of cattle, including the study of recombination features across multiple cattle breeds with high statistical power. Using the large cattle genomic database maintained by AGIL and the Council on Dairy Cattle Breeding $(\mathrm{CDCB})$, we have previously characterized the recombination features and their genetic control in dairy cattle. As mounting evidence has shown, meiotic recombination rates respond to both intrinsic and extrinsic conditions. Therefore, this study aims to determine how recombination rates vary in relation to advancing maternal age and common environment factors, such as temperature.

\section{RESULTS AND DISCUSSION}

\section{Identification of Recombination Events in Genotyped Cattle Pedigree}

Using a method developed in our previous studies (Ma et al., 2015; Wang et al., 2016), we identified recombination events by constructing three-generation families from a large cattle pedigree that included an offspring, parents, and grandparents. We phased the SNP genotypes of the focal offspring and its parents within each three-generation family. We then inferred maternal crossover events by comparing phased genotypes between dam-offspring pairs. To ensure optimal data quality, we excluded the X chromosome and used the SNP coordinates that have been corrected for potential mapping errors (Null et al., 2019; Rosen et al., 2020). In total, we extracted 305,545 threegeneration families and identified 6,677,618 maternal crossover events. All the animals have birth dates available, so we used the age of parent at birth of the focal offspring to study the effect of maternal age. Farm location and temperature information 
were available for 36,999 parents, which were included in the temperature effect analysis.

\section{Effects of Maternal Age on Recombination Rate in Cattle}

Previous studies have suggested a relationship between maternal age and the number of recombination events in plants, mice, and humans. However, even within the same species, the direction of this correlation remains inconsistent. To investigate how recombination rates are related to maternal age in cattle, we first modeled this relationship with a continuous variable of maternal age and the recombination rate residuals in cattle (Figure 1A). The recombination rate residuals were obtained by adjusting recombination rates with SNP chip density and the number of informative SNP markers within each of the 305,545 three-generation families. As a result, we observed a quadratic trend where recombination rate initially decreased from 20 to 65 months old parents and then increased as the cow grew older than 65 months. Note that we have more statistical support for the decreasing trend of recombination rate from 20 to 65 months since it consists of $91.8 \%$ of our records with much smaller standard errors. Parents that gave birth between 65 and 100 months old consist of 21,798 (7.1\%) cases of our data, and we only have 3,321 (1.1\%) cows giving birth above 100 months age. Still, the increasing trend after 65 months of maternal age is supported with a reasonable sample size $(>25,000)$. This increasing trend of recombination rate in older parents is consistent with maternal age's positive effect on recombination rate in the latest multicohort human study (Martin et al., 2015).

Since 65 months is the age that separated two groups of cows by the direction of maternal age effect, we divided the cows into ten age groups starting from 20 months old with an increment of 10 months and plotted the relationship (Figure 1B). Consistently, the same quadratic trend was observed when using maternal age as groups. Note that the last age group consists of all the records of parents giving birth over 120 months of age. To the best of our knowledge, this is the first such study in a mammalian species that reported a U-shaped relationship between maternal age and recombination rate. However, a quadratic relationship was also identified for the effect of temperature on the recombination rates in plants, fruit flies, and grasshoppers (Church and Wimber, 1969; Phillips et al., 2015), although the underlying mechanisms of meiotic recombination are completely different between cattle and these species. And this increasing of recombination after 65 months of age can also be due to culling and data representation issues because only the most fertile cows can survive on a farm for more than 65 months.

\section{Effects of Temperature on Maternal Recombination Rates in Cattle}

Previous studies have shown that temperature affects meiotic recombination rates in many poikilothermic organisms, including yeast, plants, worms, grasshoppers, and large reptiles such as crocodiles (Church and Wimber, 1969; Isberg et al., 2006; Phillips et al., 2015). However, the direction of the effect remains inconclusive. Utilizing the extensive cattle pedigree data from the United States National Cooperators Database, we characterized meiotic recombination features in dairy cows and integrated them with the environmental temperature information. Using the NOAA National Weather Database, we obtained temperature data for the months when the calves were conceived by the parents of interest. In total, we have 36,999 records with both the environmental temperature and recombination rate data.
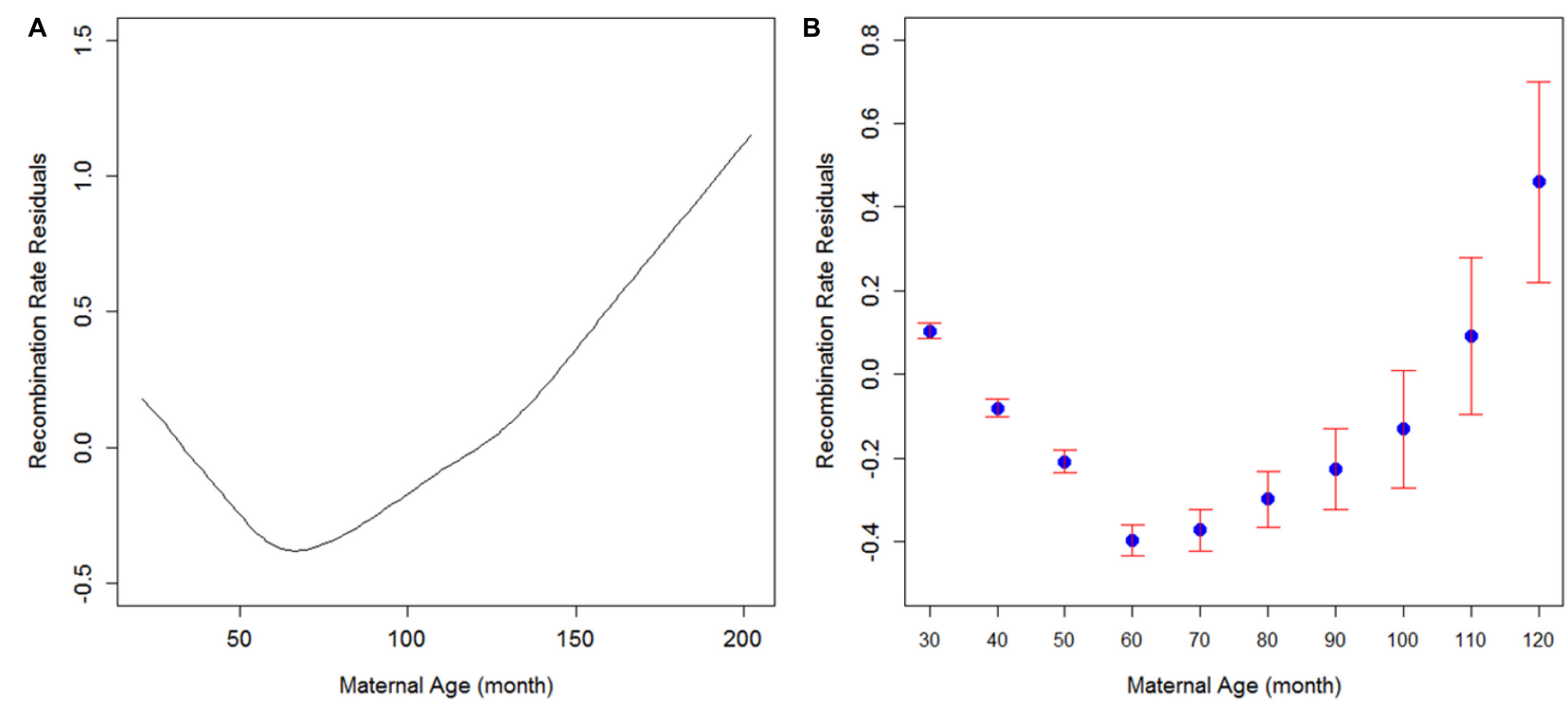

FIGURE 1 | Trend of recombination rate residuals against maternal age in cattle. (A) Fitted smooth spline of recombination rate residuals along with maternal age. The smooth spline was fitted in $\mathrm{R}$ using the smooth spline function between recombination rate residuals and maternal age. (B) Recombination rate residuals in different maternal age groups. Blue dots are means, and bars are standard errors. 
We fitted a model to explore the temperature effect on maternal recombination rates in cattle. THI (temperature humidity index) has been widely used to indicate heat or cold stress in cattle (Gaughan et al., 2008). An environment with THI exceeding 78 can be considered as a heat stress condition for cattle because both the productive and reproductive performance of cows would be seriously affected (Bohmanova et al., 2007). There is no predetermined THI index for cold stress conditions as cattle's wellness in a cold environment depends on several factors such as management practices and their hair coats. Based on the THI index in cattle, we chose two temperatures as the threshold of hot and cold conditions. Temperatures above $26.67^{\circ} \mathrm{C}$ were considered as hot conditions and temperatures below $4.44^{\circ} \mathrm{C}$ as cold conditions. In this study, we tested the effect of temperature rather than THI on recombination rate as most of previous studies reported the effect of temperature rather than THI (Church and Wimber, 1969; Loidl, 1989; Phillips et al., 2015). Still, it is interesting to investigate whether THI may have a larger impact than temperature only on recombination in future studies.

The cows were divided into three groups based on the temperature condition during the fetal development of the offspring. Over $6 \mathrm{~K}$ cows were conceived under hot conditions, over $25 \mathrm{~K}$ cows were conceived in a mild temperature environment, and $6 \mathrm{~K}$ cows were conceived under cold environment. To characterize the effect of this temperature, we reported box plots of the recombination rate residuals against those three conditions (Figure 2). We observed that cows under hot temperatures during pregnancy showed an elevation of recombination rate while cows under cold conditions showed decreased recombination rate. An increase of recombination events under hot environment is consistent with many previous studies across several species, which found an increase in recombination frequency under heat stress conditions (Lim et al., 2008; Jackson et al., 2015; Modliszewski and Copenhaver, 2017; Arrieta et al., 2021). However, the temperature effect identified here was for the fetal development stage of the offspring, instead of the female parent. Note that it is the fetal development stage of the female parent that is crucial for meiotic recombination in mammals. Therefore, the temperature effect reported here could possibly be due to an indirect effect on the fitness of the offspring with more or less crossovers, rather than on the meiosis process itself. Finally, the reported temperature effect can be explained by the 'production line' hypothesis for female recombination (Henderson and Edwards, 1968; Kong et al., 2004).

\section{Full Model Analysis of Recombination With Genetics, Maternal Age, and Temperature}

To fully understand the effect of genetic and non-genetic factors on recombination, we fitted a full model on recombination rate with all relevant factors available in our data. We modeled the genetic or animal effect as a random effect and temperature

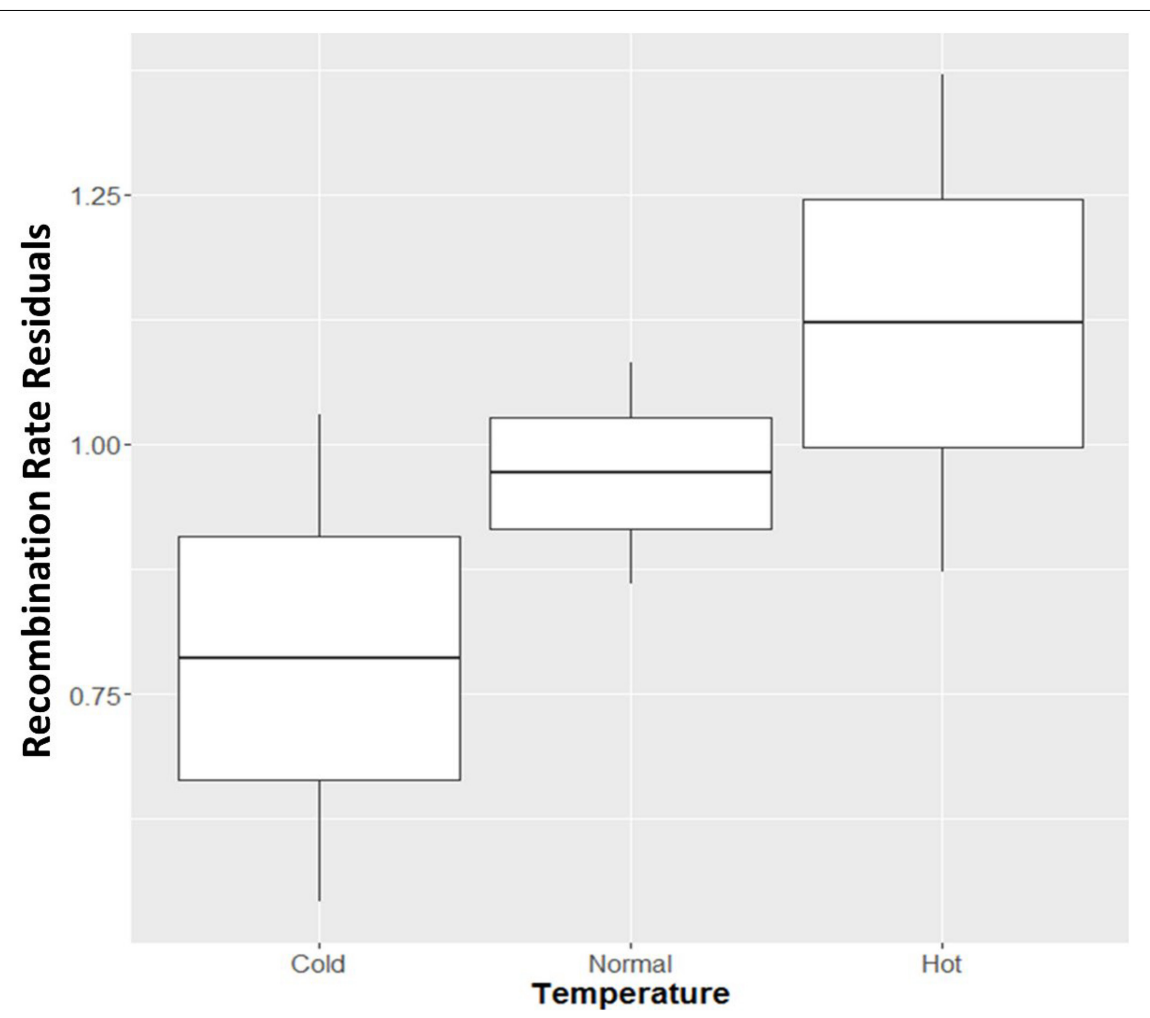

FIGURE 2 | Boxplot of recombination rate residuals in three temperature conditions during the fetal development of offspring. Cold: temperatures below $4.44^{\circ} \mathrm{C}$; Normal: temperatures between 4.44 and $26.67^{\circ} \mathrm{C}$; Hot: temperatures above $26.67^{\circ} \mathrm{C}$. 
TABLE 1 | Results of the mixed model analyses of eight factors related to the recombination rates in cattle.

\begin{tabular}{lcc}
\hline Factor & Beta & $\boldsymbol{P}$-value \\
\hline $\begin{array}{l}\text { Cold temperature during fetal development of } \\
\text { offspring }\end{array}$ & -0.194 & 0.019 \\
$\begin{array}{l}\text { Hot temperature during fetal development of } \\
\text { offspring }\end{array}$ & 0.167 & 0.027 \\
$\begin{array}{l}\text { Cold temperature during fetal development of } \\
\text { parent }\end{array}$ & 0.139 & 0.097 \\
$\begin{array}{l}\text { Hot temperature during fetal development of } \\
\text { parent }\end{array}$ & 0.093 & 0.271 \\
$\begin{array}{l}\text { Maternal Age } \\
\text { Maternal Age }\end{array}$ & -0.082 & $4.83 \times 10^{-12}$ \\
Parent Birth Year & $4.69 \times 10^{-4}$ & $5.68 \times 10^{-4}$ \\
Parent Birth Year ${ }^{2}$ & $5.02 \times 10^{-3}$ & $1.51 \times 10^{-31}$ \\
\hline
\end{tabular}

condition as a fixed effect with three levels. We included the temperatures during two important developmental stages of a female meiosis, one during the fetal development for the offspring (first generation in a three-generation family) and the other during the fetal development of the parent (second generation). Finally, we also included maternal age, the parent's birth year, and the quadratic term of these factors in the model (Table 1). Based on the genetic effect, we estimated the recombination rate's heritability to be $10 \%(S E=0.03)$, consistent with other studies in livestock animals (Sandor et al., 2012; Johnston et al., 2016; Zhang et al., 2020). Our results from this full model showed that hot temperature during the fetal development of the offspring would increase recombination rate $(P=0.027)$, while cold conditions were associated with decreased recombination rate $(P=0.019)$. However, the temperature (hot and cold conditions) during the fetal development of parents were not significantly associated with recombination rate $(P=0.271$ and $P=0.097$, respectively), although that is when female meiosis arrests. We also found that maternal age has a negative effect on recombination rates $\left(P=4.83 \times 10^{-12}\right)$ with a significant quadratic term $\left(P=5.68 \times 10^{-4}\right)$, confirming the U-shaped relationship between maternal age and recombination rate. We also noticed that a parent's birth year would positively influence recombination rates $\left(P=1.51 \times 10^{-31}\right)$ with a significant quadratic term $\left(P=1.01 \times 10^{-40}\right)$, indicating either an increasing trend of recombination rate in the dairy cattle population or some inherent confounding in the data.

\section{Potential Application of Recombination to Animal Breeding}

Theoretically, recombination should be beneficial for the longterm efficiency of selection through increasing genetic variation (Otto and Barton, 2001). However, in a short-term period, recombination may also break the combination of beneficial alleles in the haplotypes that were selected for breeding. Recent simulation studies have shown that the effect of modifying recombination rate on the improvement of traditional genomic selection is small (Battagin et al., 2016). Still, our recent work on gamete variance provided another way of using recombination on short term selection, especially for bull sires and bull dams
(Santos et al., 2019). The quadratic effect of maternal age on recombination rate suggests that young bull dams with higher recombination rate will have larger gametic variance and a better chance of producing eggs with extreme genetic merit. Finally, recombination rate does not need to be included as an independent trait in selective breeding because it has no direct economic values. But it will be under indirect, positive selection when breeding program is effective and proper selection indices used because of the long-term benefit of recombination in promoting genetic and gametic variations.

\section{CONCLUSION}

It has been shown that recombination rate can fluctuate in response to environmental changes. In this study, we used large pedigree data of dairy cattle to test the association between recombination rate and genetic and non-genetic factors, including maternal age and temperature. We discovered a non-linear association between maternal age and recombination rate in cattle, which has not been described before. Additionally, we found elevated recombination rates with increasing environmental temperature during conception. Taken together, our study provides clear evidence of an association between meiotic recombination with the non-genetic factors of maternal age and temperature. These results reveal useful insights into both the intrinsic and extrinsic effects on meiotic recombination.

\section{MATERIALS AND METHODS}

\section{Estimation of Recombination Rate in Cattle Pedigree}

We used an approach similar to the one that was developed in previous studies (Ma et al., 2015; Shen et al., 2018). First, we identified recombination/crossover events in genotyped cattle pedigree data from the national dairy genomic database hosted at the Council on Dairy Cattle Breeding (CDCB). Based on the millions of animals with genotype and pedigree data available, we extracted 305,545 three-generation families where an offspring (first generation), at least one parent (second generation), and at least one grandparent (third generation) were genotyped. We then phased the two haplotypes of an animal (first and second generations) based on the parental genotypes. We identified crossover events by comparing haplotypes in the first and second generations. Recombination events were assigned to an interval flanked by two informative SNPs (phased heterozygote SNPs in the second generation), and we estimated recombination rate between consecutive SNPs by the average crossover numbers per meiosis. We only used three-generation families that were genotyped by at least $50 \mathrm{~K}$ SNP chips. We used the ARS-UCD 1.2 genome assembly (Rosen et al., 2020) with updated SNP coordinates $^{1}$ and removed the loci from problematic regions identified in previous studies (Null et al., 2019). We only used autosome data due to the quality issues with the sex

\footnotetext{
${ }^{1}$ https://www.animalgenome.org/repository/cattle/UMC_bovine_coordinates/
} 
chromosomes. We also removed animals with more than 45 genome-wide recombination events based on the distribution of recombination across all animals, which is close to a normal distribution with mean 23.2 and variance 98.3.

\section{Temperature Data From the NOAA Database}

The National Oceanic and Atmospheric Administration (NOAA) is an American scientific agency that focuses on the conditions of the oceans and atmosphere. It's also the largest database that contains the weather records of most United States cities since 1970s. By accessing the NOAA database using the R package "rnoaa" (Edmund et al., 2014), we extracted the weather conditions during two critical periods of a cow's development that may affect the female meiotic recombination process (Table 1). The first temperature was the average temperature in the month prior to the birthdate of the offspring that measures the fetal development environment of the offspring (the first generation in a three-generation family). The second temperature was the average temperature during the month prior to the birthdate of the parent that measures the environment during the fetal development of the parent (the second generation). We then combined the temperature data with our recombination records for our mixed model analysis. By considering both the range of temperature and data availability, we divided the original temperature data into three levels: temperatures above $26.67^{\circ} \mathrm{C}$ are considered to be "hot," temperatures below $4.44^{\circ} \mathrm{C}$ are considered as "cold," and temperatures in between are "normal."

\section{A Full Model Analysis of Genetics, Maternal Age, and Temperature}

From each of the 36,009 three-generation families, we estimated the total number of crossover events per meiosis of the female parent (second generation). We then adjusted the number of crossover events by SNP density and the number of informative markers (phased heterozygote SNPs) of each animal. We first checked the maternal age effect using a smooth spline and boxplot. The smooth spline was fitted in $\mathrm{R}$ using the smooth spline function between recombination rate residuals and maternal age. Using the recombination rate residuals as phenotypes, we also fitted a linear mixed model to test for the effect of all available factors on recombination rate using the MMAP software (O'Connell, 2013). The model equation was fitted as following,

$$
\mathbf{Y}=\alpha+\mathbf{T}_{1}+\mathbf{T}_{2}+\mathbf{A}+\mathbf{A}^{2}+\mathbf{B}+\mathbf{B}^{2}+\mathbf{g}+\varepsilon
$$

where $\mathbf{Y}$ refers to the recombination rate residuals of individuals, $\mathbf{T}_{1}$ and $\mathbf{T}_{2}$ are the fixed effects for low and high temperatures,

\section{REFERENCES}

Abdullah, M. F., and Borts, R. H. (2001). Meiotic recombination frequencies are affected by nutritional states in Saccharomy cescerevisiae. Proc. Natl. Acad. Sci. U. S. A. 98, 14524-14529. doi: 10.1073/pnas.20152 9598

Arrieta, M., Willems, G., DePessemier, J., Colas, I., Burkholz, A., Darracq, A., et al. (2021). The effect of heat stress on sugar beet
A represents a fixed effect of maternal age, $\mathbf{A}^{2}$ represents the squared effect of maternal age, $\mathbf{B}$ and $\mathbf{B}^{2}$ represents the fixed effect of parent' birth year and its square, and $\mathbf{g}$ is a random effect for the genetic or animal effect on recombination rate with $\mathbf{g} \sim N\left(0, \sigma^{2} \mathbf{G}\right)$ and $\mathbf{G}$ being a genomic relationship matrix of the individuals calculated using the approach developed by VanRaden (2008). Both the temperatures during the fetal development of the parents and offspring were tested in this model. Statistical differences were declared as significant at $P<0.05$.

\section{DATA AVAILABILITY STATEMENT}

The original contributions presented in the study are included in the article. Further inquiries can be directed to the corresponding author.

\section{ETHICS STATEMENT}

Ethical review and approval was not required for the animal study because no live animals are used.

\section{AUTHOR CONTRIBUTIONS}

LM and CM conceived the study. BS, JJ, and EF analyzed and interpreted the data. EF, BS, and LM wrote the manuscript. GL and JC contributed the tools and materials. All authors read and approved the final manuscript.

\section{FUNDING}

This project was partially supported by Agriculture and Food Research Initiative Competitive Grant nos. 2020-67015-31398 and 2021-67015-33409 from the USDA National Institute of Food and Agriculture, and the BARD Grant US-4997-17 from the USIsrael Binational Agricultural Research and Development Fund. The funders had no role in study design, data collection and analysis, decision to publish, or preparation of the manuscript. CM acknowledged funding provided by Holstein Association USA (Brattleboro, VT, United States).

\section{ACKNOWLEDGMENTS}

Council of Dairy Cattle Breeding is acknowledged for providing the access to the genotype and pedigree data used in this study.

recombination. Theor. Appl. Genet. 134, 81-93. doi: 10.1007/s00122-020-0 3683-0

Battagin, M., Gorjanc, G., Faux, A.-M., Johnston, S. E., and Hickey, J. M. (2016). Effect of manipulating recombination rates on response to selection in livestock breeding programs. Genet. Sel. Evol. 48, 1-12.

Baudat, F., Buard, J., Grey, C., Fledel-Alon, A., Ober, C., Przeworski, M., et al. (2010). PRDM9 is a major determinant of meiotic recombination hotspots in humans and mice. Science 327, 836-840. doi: 10.1126/science.1183439 
Bohmanova, J., Misztal, I., and Cole, J. (2007). Temperature-humidity indices as indicators of milk production losses due to heat stress. J. Dairy Sci. 90, 1947-1956. doi: 10.3168/jds.2006-513

Cavestany, D., el-Wishy, A. B., and Foote, R. H. (1985). Effect of season and high environmental temperature on fertility of Holstein cattle. J. Dairy Sci. 68, 1471-1478. doi: 10.3168/jds.s0022-0302(85)80985-1

Chowdhury, R., Bois, P. R., Feingold, E., Sherman, S. L., and Cheung, V. G. (2009). Genetic analysis of variation in human meiotic recombination. PLoS Genet. 5:e1000648. doi: 10.1371/journal.pgen.1000648

Church, K., and Wimber, D. E. (1969). Meiosis in the grasshopper: chiasma frequency after elevated temperature and x-rays. Can. J. Genet. Cytol. 11, 209-216. doi: 10.1139/g69-025

Edmund, H., Chamberlain, S., Ram, K., and Edmund, M. H. (2014). rnoaa: 'NOAA' Weather Data from R. R Package Version 1.3.2. Available online at: https:// CRAN.R-project.org/package=rnoaa (accessed May19, 2021).

Francis, K. E., Lam, S. Y., Harrison, B. D., Bey, A. L., Berchowitz, L. E., and Copenhaver, G. P. (2007). Pollen tetrad-based visual assay for meiotic recombination in Arabidopsis. Proc. Natl. Acad. Sci. U. S. A. 104, 3913-3918. doi: 10.1073/pnas.0608936104

Gaughan, J., Mader, T. L., Holt, S., and Lisle, A. (2008). A new heat load index for feedlot cattle. J. Anim. Sci. 86, 226-234. doi: 10.2527/jas.2007-0305

Griffin, D. K., Abruzzo, M. A., Millie, E. A., Sheean, L. A., Feingold, E., Sherman, S. L., et al. (1995). Non-disjunction in human sperm: evidence for an effect of increasing paternal age. Hum. Mol. Genet. 4, 2227-2232. doi: 10.1093/hmg/4. 12.2227

Hassold, T., and Hunt, P. (2001). To err (meiotically) is human: the genesis of human aneuploidy. Nat. Rev. Genet. 2, 280-291. doi: 10.1038/35066065

Hassold, T., Merrill, M., Adkins, K., Freeman, S., and Sherman, S. (1995). Recombination and maternal age-dependent nondisjunction: molecular studies of trisomy 16. Am. J. Hum. Genet. 57, 867-874.

Henderson, S., and Edwards, R. (1968). Chiasma frequency and maternal age in mammals. Nature 218, 22-28. doi: 10.1038/218022a0

Hunter, C. M., Robinson, M. C., Aylor, D. L., and Singh, N. D. (2016). Genetic Background, maternal age, and interaction effects mediate rates of crossing over in Drosophila melanogaster females. G3 6, 1409-1416. doi: 10.1534/g3. 116.027631

Hussin, J., Roy-Gagnon, M. H., Gendron, R., Andelfinger, G., and Awadalla, P. (2011). Age-dependent recombination rates in human pedigrees. PLoS Genet. 7:e1002251. doi: 10.1371/journal.pgen.1002251

Isberg, S. R., Johnston, S. M., Chen, Y., and Moran, C. (2006). First evidence of higher female recombination in a species with temperature-dependent sex determination: the saltwater crocodile. J. Hered. 97, 599-602. doi: 10.1093/ jhered/esl035

Jackson, S., Nielsen, D. M., and Singh, N. D. (2015). Increased exposure to acute thermal stress is associated with a non-linear increase in recombination frequency and an independent linear decrease in fitness in Drosophila. Bmc Evol. Biol. 15:175. doi: 10.1186/s12862-015-0452-8

Johnston, S. E., Berenos, C., Slate, J., and Pemberton, J. M. (2016). Conserved genetic architecture underlying individual recombination rate variation in a wild population of Soay Sheep (Ovis aries). Genetics 203, 583-598. doi: 10.1534/ genetics.115.185553

Kong, A., Barnard, J., Gudbjartsson, D. F., Thorleifsson, G., Jonsdottir, G., Sigurdardottir, S., et al. (2004). Recombination rate and reproductive success in humans. Nat. Genet. 36, 1203-1206. doi: 10.1038/ng1445

Kong, A., Thorleifsson, G., Stefansson, H., Masson, G., Helgason, A., Gudbjartsson, D. F., et al. (2008). Sequence variants in the RNF212 gene associate with genome-wide recombination rate. Science 319, 1398-1401. doi: 10.1126/ science. 1152422

Levine, R. P. (1955). Chromosome structure and the mechanism of crossing over. Proc. Natl. Acad. Sci. U. S. A. 41, 727-730. doi: 10.1073/pnas.41.10.727

Lim, J. G., Stine, R. R., and Yanowitz, J. L. (2008). Domain-specific regulation of recombination in Caenorhabditis elegans in response to temperature, age and sex. Genetics 180, 715-726. doi: 10.1534/genetics.108.090142

Lipkin, S. M., Moens, P. B., Wang, V., Lenzi, M., Shanmugarajah, D., Gilgeous, A., et al. (2002). Meiotic arrest and aneuploidy in MLH3-deficient mice. Nature Genet. 31, 385-390. doi: 10.1038/ng931
Loidl, J. (1989). Effects of elevated-temperature on meiotic chromosome synapsis in Allium-Ursinum. Chromosoma 97, 449-458. doi: 10.1007/bf00295029

Lozada-Soto, E. A., Maltecca, C., Wackel, H., Flowers, W., Gray, K., He, Y., et al. (2021). Evidence for recombination variability in purebred swine populations. J. Anim. Breed. Genet. 138, 259-273. doi: 10.1111/jbg.12510

Ma, L., O'Connell, J. R., VanRaden, P. M., Shen, B., Padhi, A., Sun, C., et al. (2015). Cattle sex-specific recombination and genetic control from a large pedigree analysis. PLoS Genet. 11:e1005387. doi: 10.1371/journal.pgen.1005387

Martin, H. C., Christ, R., Hussin, J. G., O'Connell, J., Gordon, S., Mbarek, H., et al. (2015). Multicohort analysis of the maternal age effect on recombination. Nat. Commun. 6:7846.

Modliszewski, J. L., and Copenhaver, G. P. (2017). Meiotic recombination gets stressed out: CO frequency is plastic under pressure. Curr. Opin. Plant Biol. 36, 95-102. doi: 10.1016/j.pbi.2016.11.019

Null, D., VanRaden, P. M., Rosen, B., O'Connell, J., and Bickhart, D. (2019). Using the ARS-UCD1. 2 reference genome in US evaluations. Interbull Bull. $55,30-34$.

O'Connell, J. R. (2013). "MMAP: a comprehensive mixed model program for analysis of pedigree and population data," in Proceedings of the 63th Annual Meeting of The American Society of Human Genetics (Boston, MA).

Otto, S. P., and Barton, N. H. (2001). Selection for recombination in small populations. Evolution 55, 1921-1931. doi: 10.1554/0014-3820(2001)055[1921: sfrisp]2.0.co;2

Phillips, D., Jenkins, G., Macaulay, M., Nibau, C., Wnetrzak, J., Fallding, D., et al. (2015). The effect of temperature on the male and female recombination landscape of barley. New Phytol. 208, 421-429. doi: 10.1111/nph.13548

Polani, P. E., and Jagiello, G. M. (1976). Chiasmata, meiotic univalents, and age in relation to aneuploid imbalance in mice. Cytogenet. Cell Genet. 16, 505-529. doi: 10.1159/000130668

Rose, A. M., and Baillie, D. L. (1979). The effect of temperature and parental age on recombination and nondisjunction in Caenorhabditis elegans. Genetics 92, 409-418. doi: 10.1093/genetics/92.2.409

Rosen, B. D., Bickhart, D. M., Schnabel, R. D., Koren, S., Elsik, C. G., Tseng, E., et al. (2020). De novo assembly of the cattle reference genome with single-molecule sequencing. Gigascience 9:giaa021.

Sandor, C., Li, W., Coppieters, W., Druet, T., Charlier, C., and Georges, M. (2012). Genetic variants in REC8, RNF212, and PRDM9 influence male recombination in cattle. PLoS Genet. 8:e1002854. doi: 10.1371/journal.pgen.1002854

Santos, D., Cole, J., Lawlor, T. Jr., VanRaden, P., Tonhati, H., and Ma, L. (2019). Variance of gametic diversity and its application in selection programs. J. Dairy Sci. 102, 5279-5294. doi: 10.3168/jds.2018-15971

Shen, B., Jiang, J., Seroussi, E., Liu, G. E., and Ma, L. (2018). Characterization of recombination features and the genetic basis in multiple cattle breeds. BMC Genomics 19:304. doi: 10.1186/s12864-018-4705-y

Stern, C. (1926). An effect of temperature and age on crossing-over in the first chromosome of Drosophila melanogaster. Proc. Natl. Acad. Sci. U. S. A. 12, 530-532. doi: 10.1073/pnas.12.8.530

VanRaden, P. M. (2008). Efficient methods to compute genomic predictions. J. Dairy Sci. 91, 4414-4423. doi: 10.3168/jds.2007-0980

Wang, Z., Shen, B., Jiang, J., Li, J., and Ma, L. (2016). Effect of sex, age and genetics on crossover interference in cattle. Sci. Rep. 6:37698.

Zhang, J., Kadri, N. K., Mullaart, E., Spelman, R., Fritz, S., Boichard, D., et al. (2020). Genetic architecture of individual variation in recombination rate on the $\mathrm{X}$ chromosome in cattle. Heredity 125, 304-316. doi: 10.1038/s41437-020-0341-9

Conflict of Interest: The authors declare that the research was conducted in the absence of any commercial or financial relationships that could be construed as a potential conflict of interest.

Copyright (๑) 2021 Shen, Freebern, Jiang, Maltecca, Cole, Liu and Ma. This is an open-access article distributed under the terms of the Creative Commons Attribution License (CC BY). The use, distribution or reproduction in other forums is permitted, provided the original author(s) and the copyright owner(s) are credited and that the original publication in this journal is cited, in accordance with accepted academic practice. No use, distribution or reproduction is permitted which does not comply with these terms. 